\begin{tabular}{|c|c|c|c|}
\hline Risk Predictors & Patients with VA or SCD & $\begin{array}{l}\text { Patients without } \\
\text { VA/SCD }\end{array}$ & $P$ value \\
\hline Male gender & $9 / 13(69 \%)$ & $15 / 44(34 \%)$ & 0.05 \\
\hline Age $>50$ years & $11 / 13(85 \%)$ & $20 / 44(45 \%)$ & 0.02 \\
\hline Left ventricular hypertrophy & $13 / 13(100 \%)$ & 19/44 (43\%) & 0.0002 \\
\hline Presence of LGE & $7 / 10(70 \%)$ & $12 / 38(32 \%)$ & 0.03 \\
\hline QRS duration $>120 \mathrm{~ms}$ & $7 / 13(54 \%)$ & $12 / 38(32 \%)$ & 0.03 \\
\hline Dilated left atrium & $6 / 13(46 \%)$ & 9/44 (20\%) & 0.08 \\
\hline Stage $\geq 3$ CKD & $4 / 13(31 \%)$ & $11 / 44(25 \%)$ & 0.72 \\
\hline Classical variant & 10/13 (77\%) & $30 / 44(68 \%)$ & 0.73 \\
\hline $\begin{array}{l}\text { Positive HS troponin } \\
\text { (>25 ng/L) }\end{array}$ & $8 / 8(100 \%)$ & $16 / 33(48 \%)$ & 0.01 \\
\hline BNP $>400(\mathrm{ng} / \mathrm{L})$ & 10/13 (77\%) & $14 / 42(33 \%)$ & 0.009 \\
\hline
\end{tabular}

defined risk of ventricular arrhythmia (VA) and sudden cardiac death (SCD). To-date however, there is no accepted tool for risk prediction in FD and the ESC calculator in hypertrophic cardiomyopathy specifically excludes lysosomal storage diseases. Data on the prevalence of VA and SCD are restricted to single centre studies and registry data. These have identified individual risk factors including age, QRS duration $>120$ $\mathrm{ms}$, left atrial dilatation (LA), late gadolinium enhancement on cardiac magnetic resonance (CMR) imaging (LGE), and left ventricular hypertrophy $(\mathrm{LVH})$. The aim of this study was to assess the prevalence of these risk factors in a Regional FD centre and to examine known markers associated with increased cardiac mortality in FD to comprehensively assess risk.

Methods This was a retrospective cross sectional observational study of patients with a proven diagnosis of FD (genetic and clinical markers) attending the Regional Centre for Rare Diseases in Birmingham between 2012-16. As part of routine annual assessment, patients underwent 12-lead ECG, 24 hour holter monitoring, transthoracic echocardiography and multiparametric CMR. The cohort was divided into 2 groups: 1) a high risk group defined by presence of either VA ( $\geq 3$ consecutive ventricular beats at a rate $\geq 120$ beats per minute) or SCD; 2) all other patients. In addition to the above 5 risk factors, genotype (cardiac variant $\mathrm{v}$ non-cardiac variant), high sensitivity troponin (HS Tn) and NT pro-B natriuretic peptide were included. The frequency and significance of each of these proposed risk factors was studied. Fisher's exact test was used to perform statistical analysis.

Results In total 57 patients (male gender 42\%, age mean 47 \pm 18 years.) were studied, of whom 13 patients had a documented VA and 2 patients suffered SCD. 11/13 in high risk group were on ERT ( $7 \pm 6$ years.) and $21 / 44$ in the remainder $(6 \pm 3.8$ years.). Identified risk factors and prevalence are outlined in Table 1. Male gender, age $>50$, LVH, LGE, QRS $>120$ ms were all more frequent in those with VA or SCD but risk was also associated with increased HS Tn and NT pro-BNP. The presence of a cardiac variant genotype did not appear to influence risk.
Outcome These data confirm specific demographic, electrical and structural risk factors for VA and SCD in FD, although these are also present in those without arrhythmic events. Large multi-centre prospective studies are needed to further define the relative importance of these risk factors and their potential inter-relationship.

\section{INCREMENTAL DIAGNOSTIC VALUE OF CARDIOVASCULAR MAGNETIC RESONANCE IN YOUNG ADULT SURVIVORS OF SUDDEN CARDIAC ARREST}

${ }^{1}$ Adam Tsao, ${ }^{2}$ Amrit Lota*, ${ }^{3}$ Rebecca Wassall, ${ }^{3}$ John Baksi, ${ }^{3}$ Francisco Alpendurada, ${ }^{3}$ Eva Nyktari, ${ }^{3}$ Peter Gatehouse, ${ }^{3}$ David Firmin, ${ }^{3}$ Stuart Cook, ${ }^{3}$ James Ware, ${ }^{3}$ John Cleland, ${ }^{3}$ Dudley Pennell, ${ }^{3}$ Sanjay Prasad. 'Imperial College London; ${ }^{2}$ Royal Brompton Hospital, NHLI; ${ }^{3}$ Royal Brompton Hospital

\subsection{6/heartjnl-2017-311726.49}

Background The prevalence of underlying cardiovascular disease in those who die suddenly varies with age (Finocchiaro et al 2016). Cardiovascular magnetic resonance (CMR) imaging can provide incremental in-vivo diagnostic value in those resuscitated from sudden cardiac arrest (SCA) but this practice is not yet supported by guideline recommendations.

Method CMR data from consecutive patients (2002-2016) referred within 6 months of resuscitated SCA were retrospectively reviewed. Patients aged $>40$ years of age were excluded because, for them, coronary artery disease is known to be the leading cause of SCA.

Results In total, 89 SCA survivors (mean age $28 \pm 8$ years, $54 \%$ male) underwent contrast-enhanced CMR. Of these, rhythm disturbances during resuscitation were ventricular fibrillation (90\%), ventricular tachycardia (7\%), and a nondefined shockable rhythm (3\%). The CMR study was reported as normal in $47 \%$. The most commonly reported diagnoses (see figure A) were; dilated cardiomyopathy (18\%), acute myocarditis (8\%), myocardial infarction (7\%), and hypertrophic cardiomyopathy (4\%). Late gadolinium enhancement was present in $31 \%$, including $5 \%$ of patients with an otherwise normal study. Eight patients (9\%) were known to have a cardiovascular problem prior to SCA and 18\% $(n=16)$ had new disease identified by other investigations, such as echocardiography (see figure B). For the remainder of patients, CMR identified a new diagnosis in $26 \% \quad(n=23)$ and excluded important structural abnormalities in $47 \% \quad(n=42)$. The new diagnoses by CMR were early dilated cardiomyopathy (39\%), acute myocarditis (30\%), ARVC (13\%), myocardial infarction (4\%), and hypertrophic cardiomyopathy (4\%).

Conclusion Contrast-enhanced in-vivo CMR findings in young adult survivors of SCA excluded important structural cardiac disease in $47 \%$, which is similar to the rate in post-mortem studies. CMR provided incremental diagnostic value in the identification of potentially arrhythmogenic substrates due to acute myocarditis, ARVC, and dilated cardiomyopathy that may not be diagnosed by other standard investigations. These results therefore support a role for CMR in the assessment of SCA survivors. 